\title{
Role of COVID 19 Inflammatory Markers in Rhino-Orbito- Cerebral Mucormycosis: A Case Study in Predisposed Patients at a Designated Nodal Centre
}

\author{
Nitya Goddanti ${ }^{1}$ (1) $\cdot$ Y. Mounika Reddy ${ }^{1}$ - M. Kiran Kumar ${ }^{1} \cdot$ M. Rajesh ${ }^{1}$. \\ L. Sudarshan Reddy ${ }^{1}$
}

Received: 27 July 2021 / Accepted: 31 October 2021 / Published online: 13 November 2021

(C) Association of Otolaryngologists of India 2021

\begin{abstract}
Mucormycosis is a very morbid and potentially life threatening fungal infection. Sudden emergence and rapidly increasing numbers, of an otherwise rare infection in active Corona virus disease 2019 (COVID-19) or recently recovered patients has made us question the cause for this epidemic in India. This retrospective cohort study was done at Government Ear, Nose and Throat (ENT) hospital, Hyderabad, a designated nodal centre for mucormycosis for the state of Telangana, between April 2021 and June 2021. This study included patients with mucormycosis who had a recent history of COVID-19 infection. Potential predisposing factors (diabetes mellitus, history of oxygen and corticosteroids usage) and inflammatory marker values, predominantly deranged in COVID19 infection (D-dimer and Serum Ferritin) were evaluated. A correlation between the marker values and susceptibility factors was also studied. Majority of these subjects showed elevated serum markers and had one or more of the predisposing factors for COVID-19 associated mucormycosis (CAM). A significant association was found between elevated marker values and susceptibility factors (diabetes, use of oxygen). Contrary to the popular belief, that the inadvertent use of steroids, use of industrial oxygen or elevated blood sugars caused this epidemic, our study concludes that their role is limited to affecting the extent of morbidity/mortality. B.1.1.7 and B.6.117 variants of severe acute respiratory syndrome coronavirus 2 (SARSCoV2), predominant during the second wave in India, facilitated the fungal invasion and spread by altering the gene
\end{abstract}

Nitya Goddanti

nityag24@gmail.com

1 Government ENT Hospital, Koti, Hyderabad, Telangana, India expression and inducing inflammatory and immunomodulatory changes.

Keywords Mucormycosis .

COVID-19 associated mucormycosis - COVID-19 .

D-dimer $\cdot$ Serum ferritin

\section{Introduction}

The corona virus disease 2019 (COVID-19) infection, caused by novel severe acute respiratory syndrome coronavirus 2 (SARS-CoV-2) has been showing a wide and swiftly varying clinical picture, ranging from mild upper respiratory infection to fatal pneumonia. It has also been found to be associated with various bacterial and fungal secondary infections either due to pre-existing co-morbidities or as a result of aggressive treatment of life threatening COVID pneumonia. One such severe post COVID-19 infection, that emerged as an epidemic in various parts of India was COVID associated mucormycosis (CAM). Mucormycosis, popularly known as Black Fungus, is an acute fulminant invasive fungal sinusitis caused by fungi belonging to the order Mucorales. It was once considered a rare infection affecting severely immune compromised patients with risk factors like poorly controlled diabetes mellitus, organ transplantation, iron overload, kidney failure, long term steroid and immunotherapy etc. The second wave of COVID-19 has seen a very unusual and steep rise in mucormycosis in both active and recovered patients. Various factors contributing to this sudden surge have been put forward, like, deranged blood sugars, use of systemic steroids and immunomodulators, oxygen therapy during hospitalisation and use of damp, unclean masks. We have however found a sizeable group of 
patients with, normal to mildly elevated blood sugars, short duration of steroid use and no history of hospitalisation, getting affected. This prompted us to study the role of inflammatory markers during COVID-19 infection, in facilitating this rampant infection. Mucormycosis leads to clinical morbidity and mortality via angioinvasion and thromboembolism resulting in irreversible tissue necrosis. The coagulopathy seen is of complement mediated thrombotic microangiopathies, rather than sepsis induced [1]. This leads to endothelial damage and micro vascular thrombosis. D-Dimer and serum ferritin are two of the many inflammatory markers, that are elevated during COVID-19 infection. High serum ferritin levels have been proved to facilitate fungal thriving. D-dimer on the other hand, is a fibrin degradation product, high levels of which reflect the process of active clotting or thrombus formation. Thus the role these two markers was studied to find a correlation and understand the cause for mucormycosis to evolve into an epidemic particularly after the second wave of COVID-19 in India.

\section{Materials and Methods}

\section{Study Population}

A retrospective cohort study was conducted between April 2021 and June 2021 for a period of 3 months at the Government ENT hospital, Hyderabad, India. Patients with a history of COVID-19 infection within 4 weeks of onset of symptoms of rhino-cerebrospinal-orbital mucormycosis (RCOM) were only included. This was keeping in mind the fact that the inflammatory markers that rise during COVID19 infection take a minimum of 4-5 weeks to go back to normal. Patients with active COVID-19 infection were excluded as our hospital was a nodal centre, exclusively for non COVID mucormycosis cases. Those with no previous history of COVID-19 infection and with a history of surgical debridement elsewhere were also excluded. Patients with proven fungal elements in fungal culture or histopathological examination (HPE) belonging to the mucorales family, irrespective of age and sex were included in this study. A total of 300 subjects meeting the above inclusion criteria were chosen.

\section{Study Protocol}

Evaluation at presentation included a detailed history and otorhinolaryngologic, ophthalmic and neurological examinations to assess the extent of disease. The D-dimer and Serum Ferritin values recorded between day 5-7 of COVID-19 infection were collected. Detailed medical history pertaining to diabetes mellitus to categorise the patients as chronic and recent onset diabetics, along with the history of use of steroids (oral/parental) and oxygen support during COVID-19 active period was collected. These factors are believed to be the predisposing factors for this sudden surge in mucormycosis cases. Pre-operative nasal swabs were sent for potassium hydroxide $(\mathrm{KOH})$ mount study. Computerised tomography scans of paranasal sinuses and contrast enhanced magnetic resonance imaging (CE-MRI) of paranasal sinuses, orbit and brain including T1, T2, T1 fat suppression (FS), T2 FS, T1 FS post contrast, diffusion weighted imaging (DWI) were taken to study the extent of disease and to plan the surgical management. All patients presenting with a clinical and radiological picture resembling mucormycosis were immediately taken up for radical debridement. Intra-operatively specimen from the nasal cavities and or paranasal sinuses was sent for fungal culture and HPE. Surgical management included radical sino-nasal debridement with or without additional procedures like orbital decompression, orbital exenteration, partial or bilateral inferior maxillectomy depending upon the disease extension. Postoperative management included administration of Injection Liposomal Amphotericin B (5-10 mg/kg) for 14 days followed by tablet Posaconazole $600 \mathrm{mg}$ once daily on the first day followed by $300 \mathrm{mg}$ once daily for 3 months in proven cases on mucormycosis. Patients are being followed up weekly for a period of 3 months.

\section{Statistical Analysis}

The data was analysed using SPSS software for windows version 16.0 (SPSS Inc., Chicago, IL, USA). Mean, standard deviation and the minimum and maximum values of both the markers were determined, with the values rounded off to two decimal points. The values of D-dimer and Serum ferritin means were matched with the susceptibility factors mentioned above using t test for independent variables. Outcome was considered significant if the $p$ value was less than 0.05 .

\section{Results}

A total of 300 patients were included in this study of which $222(74 \%)$ were males and $78(26 \%)$ were females (Fig. 1). The mean age of presentation was $49.32 \pm 12.43$, youngest was 25 years old and oldest 85 years. The two COVID19 inflammatory markers included in this study were D-dimer, with a mean of $671.99 \pm 52.94$ and serum ferritin with a mean of $461.31 \pm 26.38$, both of which are above the normal. The mean D-dimer in males (630.24) was found less than that of females (790.83). However, the serum ferritin mean in males (470.81) was higher than in 
Fig. 1 Showing the age-sex distribution of the patients

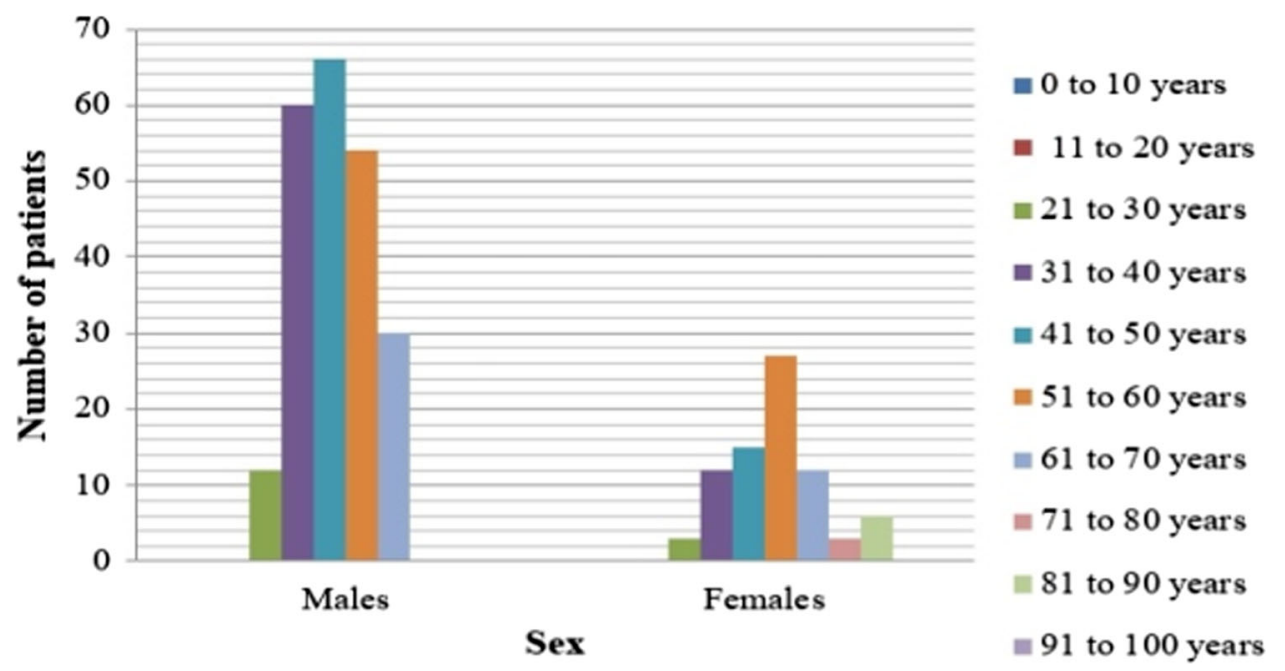

females (434.27). There was no statistically significant variation ( $p=0.18$ and $p=0.54$ respectively) of these markers based on sex. $159(53 \%)$ of the patients had D-dimer levels more than $500 \mathrm{ng} / \mathrm{ml}$ of which $48(16 \%)$ were females and $111(37 \%)$ were males. Serum ferritin values above $250 \mathrm{ng} / \mathrm{ml}$ in males and above $120 \mathrm{ng} / \mathrm{ml}$ in females was considered as elevated. In our study, a total of $231(77 \%)$ had elevated serum ferritin levels, out of which $165(55 \%)$ were males and 66 (22\%) were females (Fig. 2).

The susceptibility factors for post COVID-19 mucormycosis included in this study are, patients with diabetes mellitus (recent onset and chronic), history of steroid usage and oxygen support during COVID-19 active period. A total of $110(36.66 \%)$ had a history of recent onset diabetes mellitus and 177 (59\%) had a history of chronic diabetes. 12 (4\%) subjects were non-diabetic. A significantly large subject population 237 (79\%) had a history of oral or parental corticosteroid administration and 180 out of $300(60 \%)$ patients had a history of oxygen support during the active period. Of the 287 diabetic patients, $147(51.21 \%)$ had elevated D-Dimer values and $218(75.95 \%)$ had elevated serum ferritin values. Amongst those who had a history of corticosteroid usage (79\%), 120 $(50.63 \%)$ had elevated D-Dimer and 177 (74.68\%) had raised serum ferritin. $87(48.33 \%)$ out of 180 patients who had a history of oxygen support, had raised D-dimer and $129(71.66 \%)$ had raised serum ferritin (Fig. 3).

The inflammatory marker values were matched with the predisposing factors. It was calculated using $t$ test for independent means. Statistical significance was found between elevated means of both the inflammatory markers and history of oxygen support $(p=0.04)$. The elevated means of D-dimer and serum ferritin was also found statistically significant $(p=0.02)$ in diabetics. However, the raised mean of the markers had no statistical significance with the history of steroid usage $(p=0.07)$ in our study (Table 1).

\section{Discussion}

The second wave of COVID-19 has affected India substantially with an additional fatal threat of mucormycosis. Often referred to as 'black fungus', the incidence had risen more rapidly during the second wave compared to the first. Globally the prevalence has been as low as 0.005-1.7 per million in the past. But after COVID-19 in India alone, the prevalence has gone up to 1.4 per thousand [2, 3]. A
Fig. 2 Showing the number of patients with elevated D-dimer and serum ferritin levels

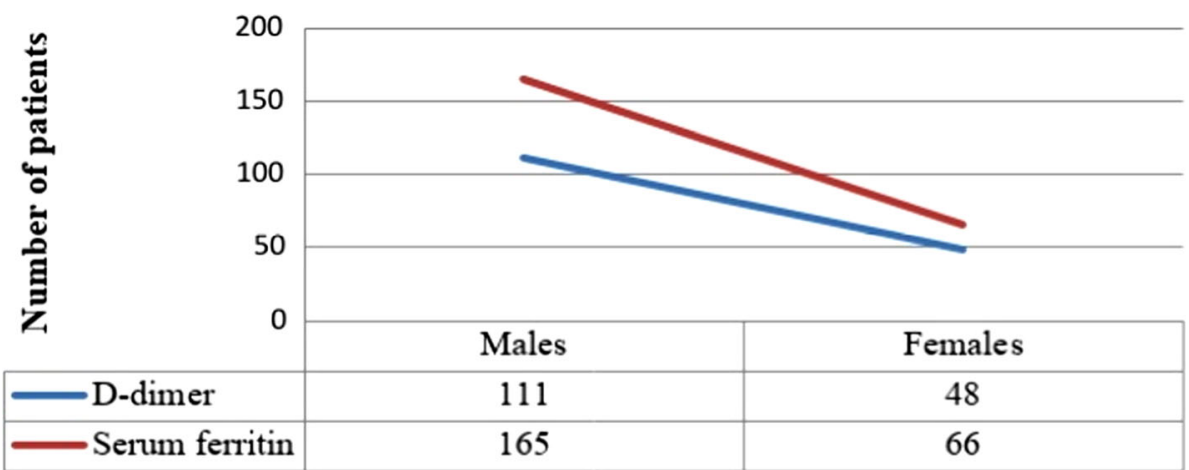


Fig. 3 Showing the distribution of patients with elevated market levels according to susceptibility factors

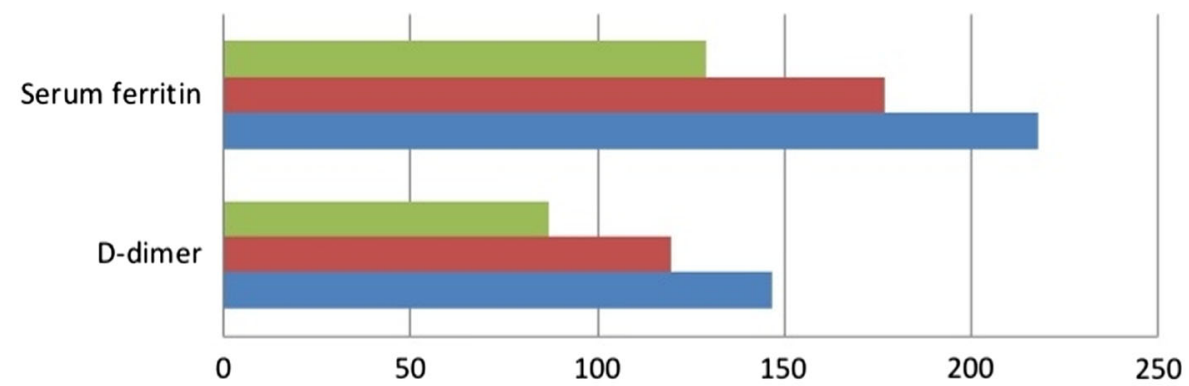

\begin{tabular}{|c|c|c|}
\cline { 2 - 3 } \multicolumn{1}{c|}{} & D-dimer & Serum ferritin \\
\hline Oxygen & 87 & 129 \\
\hline Steroids & 120 & 177 \\
\hline Diabetes & 147 & 218 \\
\hline
\end{tabular}

Table 1 Showing the statistical significance between the mean values of inflammatory markers, sex distribution and susceptibility factors using $\mathrm{t}$ test for independent variables

\begin{tabular}{|c|c|c|c|c|c|}
\hline \multirow[b]{2}{*}{ Males } & \multicolumn{2}{|c|}{ D-dimer (mean) } & \multicolumn{2}{|c|}{ Serum ferritin (mean) } & \multirow[t]{2}{*}{$p$ value } \\
\hline & 630.24 & $\mathrm{t}=1.33 p=0.18$ & 470.81 & $\mathrm{t}=-0.61 p=0.54$ & \\
\hline Females & 790.83 & & 434.27 & & \\
\hline Steroids taken & 772.21 & & 567.71 & & $\mathrm{t}=2.25 p=0.07$ \\
\hline Steroids not taken & 644.93 & & 433.03 & & \\
\hline Oxygen support present & 694.89 & & 545.20 & & $\mathrm{t}=2.87 p=0.04$ \\
\hline Oxygen support absent & 656.73 & & 405.31 & & \\
\hline Diabetes mellitus present & 787.57 & & 505.48 & & $\mathrm{t}=4.18 p=0.02$ \\
\hline Diabetes mellitus absent & 677.13 & & 459.47 & & \\
\hline
\end{tabular}

$\mathrm{t}$ (t statistic), $p$ value less than 0.05 considered significant

complex interplay of factors, including diabetes, use of immunosuppressive therapy and systemic immune alterations (reduced $\mathrm{T}$ lymphocytes altering innate immunity) of COVID-19 infection itself may have led to secondary infections like mucormycosis [4]. Approximately 25,000 cases have been reported in India over a period of around 3 months [5]. Our hospital alone saw close to 900 cases in this period. Mucormycosis is an invasive fungal infection caused by fungal species belonging to subphylum Mucormycotina, order Mucorales [6, 7]. On the basis of anatomical site of involvement, various clinical forms have been reported. Rhino-orbito-cerebral mucormycosis is the commonest form (45-74\%), followed by cutaneous (10-31\%), pulmonary (3-22\%), renal $(0.5-9 \%)$, gastrointestinal (2-8\%), and disseminated infections (0.5-9\%) [8]. Fatal Rhino-orbito-cerebral (ROCM) mucormycosis can be initiated by inhalation of fungal spores. In immunologically competent hosts, these spores will be limited by a phagocytic response [9]. If this response fails, germination will follow and hyphae will develop. Hallmarks of disease progression include angioinvasion and tissue necrosis by these fungal hyphae, that aid in fungal dissemination through the blood stream, resulting in poor penetration of anti fungal agents to the site of infection. Therefore radical surgical debridement is the first line of treatment followed by high dose anti fungal therapy.

Diabetes mellitus tends to change the normal immunological response of body to any infection in several ways. Hyperglycaemia stimulates fungal proliferation and also causes decrease in chemotaxis and phagocytic efficiency. In the diabetic ketoacidosis, there is an increased risk as these organisms produce the enzyme ketoreductase, which allows them to utilise the patient's ketone bodies and flourish. In our study $36.66 \%$ had recent onset diabetes whereas $59 \%$ had chronic diabetes and $4 \%$ were non diabetics. Rimesh Pal et al. [10] reported $85 \%$ cases with diabetes mellitus in COVID-19 associated mucormycosis. Deepak Garg et al. in their study found diabetes mellitus to be the most common risk factor [11].

Oxygen, due to use of unclean humidifiers and industrial (non-medical) oxygen, was believed to be another causative factor. $60 \%$ of our subjects had a history of oxygen 
support during COVID-19 active period. Mainak Banerjee et al. found the unforeseen use of industrial oxygen during the second wave in India to address the lack of oxygen supply for medical use as a potential cause for the blow out of CAM [12]. Improper sanitisation of oxygen cylinders, lack of clean and distilled water in oxygen humidifiers, over zealous use of steam inhalation and non humidified oxygen and presence of damp environment were put forth as some the possible precipitating factors for CAM.

Another predominant risk factor proposed was the injudicious use of corticosteroids in COVID-19 cases. Many active cases with mild symptoms or without hypoxemia had a history of prolonged use of higher doses of corticosteroids [12]. Current guidelines in India recommend $0.5 \mathrm{mg} / \mathrm{kg} /$ day body weight of methylprednisolone (parenteral) for three days in moderate cases and $1-2 \mathrm{mg} / \mathrm{kg} /$ day in severe cases [13]. The National Institute of Health recommends the use of Dexamethasone, $6 \mathrm{mg} /$ $\mathrm{kg} / \mathrm{day}$ for a maximum of 10 days, in patients requiring ventilator or supplemental oxygen [14]. The guidelines specifically mention the risk of secondary infections. White $P L$ et al. [15] stated that corticosteroid therapy during COVID 19, was associated with higher risk of invasive fungal sinusitis. The propensity of corticosteroids to impair migration, ingestion and phagolysosome fusion in macrophages may explain suppressed immunity in such patients [16]. However, a sizeable number of patients with history of short course of low dose corticosteroids, diabetes with controlled blood sugars and with a history of home isolation were also seen to have developed mucormycosis in our study. This prompted us to study the possible role of deranged inflammatory markers caused as a result of SARS $\mathrm{CoV} 2$, in triggering CAM.

Two of the many COVID-19 markers routinely tested during active phase included in this study are, D-dimer and serum ferritin. D-dimer is a fibrin degradation product, a small protein fragment present in blood after a clot is degraded by fibrinolysis. The role of $\mathrm{D}$-dimer in mucormycosis was thought of during the course of debridement surgeries at our centre, wherein, very unusual and frequent clotting of blood was noted intraoperatively. This gave us a suspicion of the role of abnormal thromboembolism along with fungal invasion leading to tissue necrosis. Our study showed a mean D Dimer range of $671.99 \pm 52.94$ which is higher than normal $(\leq 500 \mathrm{ng} /$ $\mathrm{ml}$ ). Raised serum iron levels is an already established risk factor for mucormycosis. Several patients with COVID-19 infection showed elevated serum ferritin levels resulting due to cytokine storm and secondary hemophagocytic lymphohistiocytosis. Patients with elevated levels free iron (not bound to transferrin) are uniquely susceptible to infection by Rhizopus oryzae and other Zygomycetes, but not to other pathogenic fungi, such as candida or aspergillosis [17, 18]. Patients with diabetic ketoacidosis (DKA) have elevated levels of available serum iron, likely due to release of iron from binding proteins in the presence of acidosis [19]. Free iron is toxic, hence it's utilisation by microorganisms is limited. Fungi of Zygomycetes group on the other hand show increased uptake and storage by reduction of ferric to ferrous form and storage as a part of siderophores $[20,21]$. Serum ferritin levels $\leq 250 \mathrm{ng} / \mathrm{ml}$ (in males) and $\leq 120 \mathrm{ng} / \mathrm{ml}$ (in females) are considered normal. The mean serum ferritin level in our study is $461.31 \pm 26.38$ which is above normal for either sex.

The elevated means of D-dimer and serum ferritin levels were found to have a significant association with coexisting diabetes mellitus and use of oxygen during active period in our study. However, there was no literature found to have studied the interrelationship between the said predisposing factors and COVID-19 inflammatory markers in CAM. Interestingly, there were sizeable number of subjects who had no history of corticosteroid usage, oxygen support and with controlled blood sugars who developed CAM. This raised the suspicion about the role of SARS CoV 2 (B.1.1.7 and B.6.117) variant that led to second wave in the sudden rise of these cases [22, 23]. It was well established that the virus gains entry into cells using angiotensin converting enzyme -2 (ACE-2) receptors. Ibrahim et al. [17] and others have reported that glucose related protein 78 (GRP78) could act as an alternative route for virus entry. It is noted mucor fungus also has the same port of entry i.e., GRP78 into nasal and paranasal sinus mucosa through its coat protein spore coat protein homologue (CotH3 [24]. In COVID-19 infection there is an increased expression of GRP78 making the cells vulnerable to fungal pathogens $[17,25]$. This could explain both the higher transmissibility of SARSCoV2 and surge in CAM.

\section{Conclusion}

COVID-19 pandemic has shown a myriad of clinical variations in a short span of time. One such variation, that evolved into an epidemic in itself, is COVID-19 associated Rhino-orbito-cerebral mucormycosis, in India. Several theories for this sudden rise of an otherwise rare fungal secondary infection have been put forward which included the use of corticosteroids, co-morbidities like diabetes mellitus, history of oxygen support etc. However, it is when the subjects without these predisposing factors started presenting with CAM that, the role of SARSCoV2 in the pathogenesis came into light. Our study concludes that, though majority of the subjects have had one or more the above mentioned predisposing factors, the role of elevated D-dimer coupled with the use of oxygen and elevated serum ferritin levels coupled with use of oxygen and 
corticosteroids is significant. Having said that, it appears that the virus induced vulnerability is the primary cause for this epidemic. The role of these predisposing factors seems to be limited to modulate the severity and extent of the disease and it's morbidity.

Acknowledgements We would like to thank all our patients for their cooperation, our medical superintendent Dr. T Shankar for leading us through this unforeseen challenge, for motivating and encouraging us to conduct this study and Subramanyam Dharba (M.S. Epidemiology, Public Health) Consultant Biostatistician for helping us with the statistics.

Authors' Contribution All authors contributed to the study conception and design. Material preparation, data collection and analysis were performed by Nitya Goddanti, Y Mounika Reddy, Kiran Kumar M, M Rajesh and L Sudarshan Reddy. The first draft of the manuscript was written by Nitya Goddanti and all authors commented on previous versions of the manuscript. All authors read and approved the final manuscript.

Funding The author(s) received no financial support for the research, authorship and/or publication of this article.

\section{Declarations}

Conflict of interest The author(s) declared no potential conflicts of interest with respect to the research, authorship, and/or publication of this article.

Ethical Approval The study protocol was approved by the institution ethics committee.

Informed Consent As this was a retrospective analysis of medical charts, no informed written consents were taken.

\section{References}

1. Campbell CM, Kahwash R (2020) Will complement inhibition be the new target in treating COVID-19-related systemic thrombosis? Circulation 141:1739-1741. https://doi.org/10.1161/ CIRCULATIONAHA.120.047419

2. Petrikkos G, Skiada A, Lortholary O, Roilides E, Walsh TJ, Kontoyiannis DP (2012) Epidemiology and clinical manifestations of mucormycosis. Clin Infect Dis 54(SUPPL. 1):S23-S34. https://doi.org/10.1093/cid/cir866

3. Chander J, Kaur M, Bhalla M, Punia RS, Singla N, Bhola K, Alastruey-Izquierdo A, Stchigel AM, Guarro J (2015) Changing epidemiology of mucoralean fungi: chronic cutaneous infection caused by mucor irregularis. Mycopathologia 180:181-186. https://doi.org/10.1007/s11046-015-9908-Z

4. Chen N, Zhou M, Dong X et al (2020) Epidemiological and clinical characteristics of 99 cases of 2019621 novel coronavirus pneumonia in Wuhan, China: a descriptive study. Lancet (London, England) 395(10223):507-13

5. Akshay R, Huy NT (2021) Rising incidence of mucormycosis in patients with COVID-19: another challenge for India admits the second wave. Lancet Respiratory Med. https://doi.org/10. 1016/S2213-2600(21)00265-4

6. Ribes JA, Vanover-Sams CL, Zygomycetes BDJ (2002) In human disease. Clin Microbiol Rev 13:236-301
7. Spellberg B, Edwards J Jr, Ibrahim A (2005) Novel perspectives on mucormycosis: pathophysiology, presentation, and management. Clin Microbiol Rev 18(3):556-569. https://doi.org/10.11 28/CMR.18.3.556-569.2005

8. Priya P, Ganesan V, Rajendran T, Geni VG (2020) Mucormycosis in a tertiary care center in South India: a 4-year experience. Indian J Crit Care Med Peer-Reviewed Off Publ Indian Soc Crit Care Med 24(3):168-171. https://doi.org/10.5005/jp-journals10071-23387

9. Mignogna M, Fortuna G, Leuci S, Adamo D, Ruoppo E, Siano M, Mariani U (2011) Mucormycosis in immunocompetent patients: A case-series of patients with maxillary sinus involvement and a critical review of the literature. Int J Infect Dis IJID Off Publ Int Soc Infect Dis 15:e533-e540. https://doi.org/10.1016/ j.ijid.2011.02.005

10. Pal R, Banerjee M, Yadav U, Bhattacharjee S (2020) Clinical profile and outcomes in COVID-19 patients with diabetic ketoacidosis: a systematic review of literature. Diabetes Metab Syndr 14:1563-1569

11. Garg D, Muthu V, Sehgal IS et al (2021) Coronavirus disease (Covid-19) associated mucormycosis (CAM): case report and systematic review of literature. Mycopathologia 186:289-298. https://doi.org/10.1007/s11046-021-00528-2

12. Banerjee M, Pal R, Bhadada SK (2021) Intercepting the deadly trinity of mucormycosis, diabetes and COVID-19 in India. Postgrad Med J. https://doi.org/10.1136/postgradmedj2021-140537

13. Clinical management protocol: COVID-19, Directorate general of health services (EMR), ministry of health and family welfare, government of India; Version 3, 13.06.20

14. RECOVERY Collaborative Group, Horby P, Lim WS, Emberson JR, Mafham M, Bell JL, Linsell L, Staplin N, Brightling C, Ustianowski A, Elmahi E, Prudon B, Green C, Felton T, Chadwick D, Rege K, Fegan C, Chappell LC, Faust SN, Jaki T, Landray MJ (2021) Dexamethasone in hospitalized patients with Covid-19. N Engl J Med 384(8):693-704. https://doi.org/ 10.1056/NEJMoa2021436

15. White PL, Dhillon R, Cordey A, Hughes H, Faggian F, Soni S, Pandey M, Whitaker H, May A, Morgan M, Wise MP, Healy B, Blyth I, Price JS, Vale L, Posso R, Kronda J, Blackwood A, Rafferty H, Moffitt A, Backx M (2020) A national strategy to diagnose COVID-19 associated invasive fungal disease in the ICU. Clin Infect Dis Off Publ Infect Dis Soc Am. https://doi.org/10.1093/cid/ciaa1298

16. Jose A, Singh S, Roychoudhury A et al (2021) Current understanding in the pathophysiology of SARS-CoV-2-associated rhino-orbito-cerebral mucormycosis: a comprehensive review. J Maxillofac Oral Surg. https://doi.org/10.1007/s126 63-021-01604-2

17. Ibrahim AS, Spellberg B, Edwards J Jr (2008) Iron acquisition: a novel perspective on mucormycosis pathogenesis and treatment. Curr Opin Infect Dis 21(6):620-625. https://doi.org/10.1097/ QCO.0b013e3283165fd1

18. Sugar AM (1992) Mucormycosis. Clin Infect Dis 14:S126-S129

19. Artis WM, Fountain JA, Delcher HK, Jones HE (1982) A mechanism of susceptibility to mucormycosis in diabetic ketoacidosis: transferrin and iron availability. Diabetes 31:1109-14

20. Matzanke BF, Winkelmann G, Winge DR (1994) Metal ions in fungi, vol 11. Marcel Dekker Inc., pp 179-214

21. Dix DR, Bridgham JT, Broderius MA, Byersdorfer CA, Eide DJ (1994) The FET4 gene encodes the low affinity Fe(II) transport protein of Saccharomyces cerevisiae. J Biol Chem 269:26092-26099

22. Morales-Franco B, Nava-Villalba M, Medina-Guerrero EO et al (2021) Host-pathogen molecular 589 factors contribute to the 
pathogenesis of Rhizopus spp. Diabetes Mellitus Curr Trop Med Rep 590(2021):1-12

23. Vaidyanathan G (2021) Coronavirus variants are spreading in india-what scientists know so far (news in focus). Nature 593:321-322

24. Elfiky AA, Ibrahim IM, Elgohary AE (2021) Host-cell recognition through GRP78 is enhanced in the new variants of SARSCoV-2; in silico perspective. Res Sq. https://doi.org/10.21203/rs. 3.rs- $189975 / \mathrm{v} 1$
25. Allam L, Ghrifi F, Mohammed H (2020) Targeting the GRP78dependant SARS-CoV-2 cell entry by peptides and small molecules. Bioinform Biol Insights 14:1177932220965505. https://doi.org/10.1177/1177932220965505

Publisher's Note Springer Nature remains neutral with regard to jurisdictional claims in published maps and institutional affiliations. 